\title{
On the origin of solar wind. Alfvén waves induced jump of coronal temperature
}

\author{
T.M. Mishonov * M.V. Stoev $\oplus^{\dagger}$ and Y.G. Manevd \\ Department of Theoretical Physics, Faculty of Physics, \\ University of Sofia St. Clement of Ohrid, \\ 5 J. Bourchier Boulevard, Bg-1164 Sofia, Bulgaria
}

(Dated: August 16, 2018)

\begin{abstract}
Absorbtion of Alfvén waves is considered to be the main mechanism of heating in the solar corona. It is concluded that the sharp increase of the plasma temperature by two orders of magnitude is related to a self-induced opacity with respect to Alfvén waves. The maximal frequency for propagation of Alfvén waves is determined by the strongly temperature dependent kinematic viscosity. In such a way the temperature jump is due to absorption of high frequency Alfvén waves in a narrow layer above the solar surface. It is calculated that the power per unit area dissipated in this layer due to damping of Alfvén waves blows up the plasma and gives birth to the solar wind. A model short wave-length (WKB) evaluation takes into account the $1 / f^{2}$ frequency dependance of the transversal magnetic field and velocity spectral densities. Such spectral densities agree with old magnetometric data taken by Voyager 1 and recent theoretical calculations in the framework of Langevin-Burgers MHD. The present theory predicts existence of intensive high frequency MHD Alfvén waves in the cold layer beneath the corona. It is briefly discussed how this statement can be checked experimentally. It is demonstrated that the magnitude of the Alfvén waves generating random noise and the solar wind velocity can be expressed only in terms of satellite experimental data. It is advocated that investigation of properties of the solar surface as a random driver by optical methods is an important task for future solar physics. Jets of accretion disks are speculated as a special case of the wind from magnetized turbulent plasma.
\end{abstract}

PACS numbers: 52.35.Bj, 52.50.Sw, 96.60.P-, 94.30.cq

\section{INTRODUCTION}

The coronal heating mechanism ${ }^{1,2}$ is one of the most perplexing longstanding unresolved problems of contemporary physics. ${ }^{3}$ This is the reason why even some condensed matter theorists are involved in the revealing of this mystery. The lack of experimental data makes distinguishing between the various theoretical models difficult. In such a situation it is possible to apply purely aesthetic criteria for a natural description of some well-known facts. One such fact is that the increase of plasma temperature in the transition region by two orders of magnitude ${ }^{4}$ occurs very rapidly - there is a smeared jump, which serves as a starting point for creation of the solar wind. We wish to emphasize that complete magneto-hydrodynamic (MHD) simulations of Alfvén waves generated by a random driver also give a sudden increase of the temperature as a function of height. $\frac{5}{-}$ The purpose of the current work is to give a qualitative explanation of the observed temperature jump and to perform a model evaluation of its order of magnitude. Finally, we will analyze some possible future experimental observations described by the presented theoretical scenario. For example, the model predicts existence of intensive high frequency Alfvén waves beneath the coronal temperature jump.

\section{SCENARIO}

In order to make a proper assessment for the magnetic field spectrum comparable with magnetometric data taken by Voyager $1^{6}$ we need to switch to a frequencydependent regime and perform a time averaging of the Fourier transformed wave component $\mathbf{B}(t) \approx B_{x} \hat{x}+B_{y} \hat{y}$ perpendicular to the constant magnetic field $\mathbf{B}_{0}=B_{0, z} \hat{z}$ along which the $z$-axis is chosen

$$
\begin{aligned}
& \mathbf{B}(t)=\sum_{\omega_{n}} \exp \left(-i \omega_{n} t\right) \mathbf{B}_{\omega_{n}}, \quad \omega_{n}=\frac{2 \pi}{\Delta t} n \\
& \mathbf{B}_{\omega_{n}}=\frac{1}{\Delta t} \int_{0}^{\Delta t} \exp \left(i \omega_{n} t\right) \mathbf{B}(t) \mathrm{d} t, \quad n=0, \pm 1, \pm 2, \ldots
\end{aligned}
$$

As our purpose is to present a model evaluation, from now on we will consider only one of the fluctuating wave components, so the plane magnetic field indices will be omitted. Fourier analysis of the signal accumulated for a time interval $\Delta t$ gives us the opportunity to observe the magnetic field spectral density $\Phi(t)$, which is tightly bound to the time averaged square of the wave magnetic component

$$
\left\langle B^{2}(t)\right\rangle=\frac{1}{\Delta t} \int_{0}^{\Delta t} B^{2}(t) \mathrm{d} t=\sum_{\omega_{n}}\left|B_{\omega_{n}}\right|^{2}=\int \Phi(f) \mathrm{d} f .
$$

The thus-defined spectral density

$$
\begin{aligned}
\Phi(f) & =\frac{1}{f_{2}-f_{1}} \sum_{\omega_{n}}\left|B_{\omega_{n}}\right|^{2}, \quad \omega_{n} \in\left(\omega_{1}, \omega_{2}\right), \\
f & =\omega / 2 \pi, \quad\left(f_{2}-f_{1}\right) \Delta t \gg 1
\end{aligned}
$$

determines the magnetic field energy density $w$ and the Alfvén waves energy flux $S$ as a function of the circular 
$\omega$ or linear frequency $f$

$$
w=\frac{B^{2}(t)}{2 \mu_{0}}, \quad S=\frac{V_{\mathrm{A}}}{2 \mu_{0}} \int \Phi(f) \mathrm{d} f .
$$

In interplanetary space the spectral density $\Phi$ is measured in typical units $\mathrm{nT}^{2} / \mathrm{Hz}$. Alfvén waves energy density depends on the integrated spectral density and the Alfvén speed $V_{\mathrm{A}}$. In general the integration should be taken over all possible wave frequencies $f \in(0, \infty)$ unless the given specific power spectrum imposes an introduction of a low or high frequency cut-off. In the suggested model we use the natural cut-off frequency set by the condition for waves existence $\omega_{\mathrm{A}} \tau_{\mathrm{A}}=1$, when the Alfvén waves frequency $\omega_{\mathrm{A}}$ exactly equals the attenuation coefficient $\tau_{\mathrm{A}}{ }^{-1}$. Therefore in the investigation that follows we exclude the influence of any extremely highfrequency Alfvén waves that would be absorbed by the medium immediately after their generation under the solar surface. Hence for heating in the corona we only take into account those waves, whose characteristic life time exceeds the inverse value of the cut-off frequency $\omega_{c}$

$$
\begin{aligned}
\omega_{\mathrm{A}} \tau_{\mathrm{A}} & >1, \quad \omega_{\mathrm{A}}=V_{\mathrm{A}} k_{z}, \quad 1 / \tau_{\mathrm{A}}=\nu k^{2}, \\
\rho V_{\mathrm{A}}^{2} & =B_{0, z}^{2} / \mu_{0}, \quad \omega_{c}=2 \pi f_{c}=V_{\mathrm{A}}^{2} / \nu .
\end{aligned}
$$

Molecular kinetic theory ${ }^{\underline{7}}$ determines the temperaturedependence of the cut-off frequency, being a function of the kinematic viscosity $\nu$. A more detailed analysis shows that the sum of kinematic and magnetic viscosity is relevant in the damping of the Alfvén waves ${ }^{8.9}$, but the magnetic viscosity is much smaller and negligible for sufficiently high plasma temperatures. This is because the kinamatic viscosity is $\propto T^{5 / 2}$ while the resistivity is $\propto T^{-3 / 2}$. For more detailed discussion see, for example, the work by Erdelyi and Goossens ${ }^{10}$ and Ref. 11 . For low density coronal plasmas, electromagnetic emission and thermal conduction are negligible for the energy balance close to the temperature jump. In our model we neglect the difference between the electron and ion temperatures which are of the same order $T_{e} \approx T_{p}$. However we wish to emphasize that heating is due to ion viscosity and our consideration gives a natural explanation why the proton temperature is higher than electron one $T_{p}>T_{e}$. It is because viscous friction heats the ions. Since we pursue just a qualitative estimation, with a logarithmic accuracy in the final results for the solar wind velocity presented below, we can neglect the slight temperature variations in the protons' Coulomb logarithm $L_{p}$ as well as the influence of the electron temperature dependence on the Debye radius $a$. Precise calculations including such a dependence would only lead to minor corrections that would not change the order of magnitude of the final outcome

$$
\begin{aligned}
\nu & =c_{\nu} T_{p}^{5 / 2}, \quad c_{\nu}=\frac{0.4}{M^{1 / 2} e^{4} n_{p} L_{p}}, \quad L_{p}=\ln \frac{T_{p} a}{e^{2}} \gg 1 \\
\frac{1}{a^{2}} & =4 \pi e^{2}\left(\frac{n_{e}}{T_{e}}+\frac{n_{p}}{T_{p}}\right), \quad e^{2} \equiv \frac{q_{e}^{2}}{4 \pi \varepsilon_{0}} .
\end{aligned}
$$

According to valuable analysis on the experimental data obtained by the Voyager 1 magnetometer ${ }^{6}$, the magnetic field spectral density can be approximated by a single power law. Here we have taken into account that both magnetic and energy fluxes are almost constant along the magnetic field lines. In such a way the spectral parameter $\mathcal{D}$ on the solar surface can be evaluated by order of magnitude if we know the satellite spectral parameter $\mathcal{D}^{\text {(sat) }}$ and the ratio of the constant components of the magnetic field

$$
\Phi(f) \approx \frac{\mathcal{D}}{f^{2}}, \quad \mathcal{D} \simeq \frac{B_{0, z}}{B_{0, z}^{(\text {sat })}} \mathcal{D}^{(\text {sat })} .
$$

The observed power law for the energy density $\propto 1 / f^{2}$ is theoretically explained in the framework of LangevinBurgers MHD mode ${ }^{12}$. The 1D calculations for the time and noise averaged spectral density of Alfvén waves generated by a white noise random driver for the external force density, modeling the influence of the convective stochasticity

$$
\left\langle F\left(t_{1}, z_{1}\right) F\left(t_{2}, z_{2}\right)\right\rangle=\tilde{\Gamma} \rho^{2} \delta\left(t_{1}-t_{2}\right) \delta\left(z_{1}-z_{2}\right)
$$

reveal the same inverse proportionality to the second power of the Alfvén frequency

$$
\bar{E}_{f}=\frac{\pi^{2} \rho V_{\mathrm{A}}^{2} \tilde{\Gamma}}{2 \nu f^{2} L} .
$$

Comparison of the energy flux theoretically derived on the basis of the Langevin-Burgers approach applied for modeling the role of the turbulence for generation of Alfvén waves with the experimentally observed energy flux can give us a reliable assessment for the Burgers parameter $\tilde{\Gamma}$

$$
S=L \int V_{\mathrm{A}} \bar{E}_{f} \mathrm{~d} f=\int \frac{\pi^{2} \rho V_{\mathrm{A}}^{2} \tilde{\Gamma}}{2 \nu f^{2}} \mathrm{~d} f=\int \frac{V_{\mathrm{A}} \mathcal{D}}{2 \mu_{0} f^{2}} \mathrm{~d} f .
$$

Thus, if we consider Burgers approach as adequate for a turbulence model description, we can extract information for the turbulence spectrum in the photosphere, at the footpoints of the magnetic field lines

$$
\tilde{\Gamma}=\mathcal{D} \nu / \pi^{2} \mu_{0} \rho V_{\mathrm{A}} .
$$

According to a recently proposed scenario $\frac{12,13}{13}$ in the spirit of earlier ideas for wave heating 15,16 Alfvén waves serve as mediators, carriers of energy from the turbulent photosphere to the hot solar atmosphere, where in a small region the high-frequency waves attenuate intensively and heat the corona.Ref. $\frac{12}{}$ treats the emission, while Ref 13 considers the bulk absorption of Alfvén waves. In this work we present an evaluation for the absorbed in the transition zone energy flux, whose strong temperature dependence is determined by the temperature dependence of the cut-off frequency $f_{c}$ and naturally results in a sharp temperature jump $\underline{13}$

$$
f_{c}=\frac{V_{\mathrm{A}}^{2}}{2 \pi c_{\nu} T^{5 / 2}} .
$$


The absorbed energy flux is taken from Voyager 1 magnetometric data analysis, but it may also be obtained by a thermodynamical approach. If the comparatively small effects associated with radiative losses and compression are neglected, the absorption rate will be related only to the plasma internal energy density $\varepsilon$ and the conducted work

$$
\begin{aligned}
S_{\mathrm{Abs}} & =V_{\mathrm{A}} \int_{f_{c}}^{\infty} \frac{\mathcal{D}}{2 \mu_{0} f^{2}} \mathrm{~d} f=\frac{V_{\mathrm{A}} \mathcal{D}}{2 \mu_{0} f_{c}}=\frac{\pi c_{\nu} \mathcal{D}}{\mu_{0} V_{\mathrm{A}}} T_{p}^{5 / 2} \\
& \approx \varepsilon v+p v, \quad \varepsilon=\frac{3}{2} p, \quad p=n_{e} T_{e}+n_{p} T_{p}
\end{aligned}
$$

where $p$ and $v$ are respectively the plasma pressure and velocity. In such a way we can derive the approximate rate for the velocity of the solar wind, driven by sharp coronal temperature increase due to absorption of intensive high frequency Alfvén waves, for which the transition region plasma is opaque

$$
v_{\text {wind }} \simeq \frac{0.08 \pi \mathcal{D} T_{p}^{3 / 2}}{\mu_{0} V_{\mathrm{A}} M^{1 / 2} e^{4} n_{p}^{2} L_{p}}, \quad T_{p} \sim T_{e} .
$$

As the shear viscous friction heats the heavy particles,$\frac{10.14}{\longleftarrow}$ the proton temperature $T_{p}$ is significantly higher than that of the electrons $T_{e}$, however, for an order of magnitude evaluation here we suppose the electron temperature $T_{e}$ to be similar to that of the protons $T_{p}$.

\section{DISCUSSION AND CONCLUSIONS}

With a logarithmic accuracy we have derived an explicit formula for the initial velocity of the solar wind. This formula Eq. (14) is completely based on experimentally accessible parameters and can be easily rejected if it gives more than 3 orders of magnitude difference. But, if this model remains valid, let us briefly discuss what has to be done as a future perspective in order to finally solve the perplexing longstanding mystery for the origin of coronal heating and solar wind. First of all, numerical simulations on MHD with a white noise random driver ${ }^{5}$ (Langevin-Burgers MHD) have to be repeated to reproduce a $\delta$-like maximum of the energy dissipation density at the transition region. In other words all theoretical models qualitatively explaining the Voyager 1 data for the frequency dependent spectral density of the magnetic field have to be compared with random driver computer simulations of coronal heating. Confirmation of a narrow maximum of volume heating power due to self-induced plasma opacity is a routine task for further numerical investigations. The numerical analysis could improve the present analytical evaluation incorporating, for example, the reflection of Alfvén waves by the jump in plasma density.

Here we wish to insert a short historical remark. Stochastic mechanics in general was introduced by Langevin $\frac{17}{}$ in 1905 to explain the Brownian motion.
Later, Burgers 18 in 1948 introduced the white noise random driver in the hydrodynamics of turbulence. Much later, in 1995, Polyakov $\underline{19}$ derived Kolmogorov power laws ${ }^{20}$ using Langevin-Burgers approach, but his research remained unobserved in astrophysics, not to speak about heliophysics. That is why using random number generator in MHD simulations is simply called random driver, but the magnitude of the noise is almost never evaluated by comparison to real experimental data.

In the recent work we have only evaluated the area under the sharp maximum of dissipation and now it is time to perform state of the art calculations with a realistic random force, whose spectral density corresponds to the data taken by the satellites' magnetometers. For example, the turbulence spectrum can be treated with the based on the Langevin-Burgers model assessment Eq. (11). We propose that the physical mechanism for the origin of solar wind and coronal heating is already revealed and it is only a matter of honest numerical work to create a coherent picture. The results for the temperature jump and dissipation maximum have to be compared with the observations and other theoretical scenarios such as magnetic reconnections 21 for example. Each model unable to reproduce a sharp temperature increase in height has to be assigned to the waste basket. The same can be said for the models based on Ohmic heating which predict an electron temperature higher than that of the protons. Though the latter statement seems to be true for the X-ray bright points, it is definitely not valid for the corona as a whole. We are unaware, for example, how magnetic reconnection theory can explain why the proton temperature is higher than that of the electrons $T_{p}>T_{e}$. Analogously we have not found any reference explaining how turbulent cascade can lead to a very sharp increase of the coronal temperature.

Second, in order to evaluate the properties of the solar surface as a random driver, all the old data from Voyager 1 has to be meticulously analyzed and a detailed investigation by the forthcoming Solar Orbiter mission has to be planned.

Third, the Sun is a unique system for investigation of convective turbulence. It will be very interesting to compare the satellites' data for the magnetic field spectral density with results based on theoretical modeling of turbulence. The maturity of solar physics can stimulate significant development of the achievements in contemporary turbulence research. For instance, the $1 / f^{2}$ power law by Burlaga and Mish ${ }^{6}$ corresponds to onedimensional $\left(k_{x}, k_{y}=0\right)$ propagation of Alfvén waves in the framework of Langevin MHD $\stackrel{12}{,}$ as the whole noise is created by the random motion of the funnel foot-points.

Next, absorbption of Alfvén waves could be an important mechanism in many cases of space plasmas including accretion disks as well. MHD waves in accretion disk amplification by shear flow is the main mechanism of transformation of gravitational energy into heat; for references see the preprint $\underline{\underline{22}}$ The magnetized wind from an accretion disk should follow the magnetic force lines 
and plasma concentrated above the disk center will create an accumulative jet perpendicular to the disk. In such a way disk jets could be recognized as a wind coming from magnetized turbulent plasma.

Lastly, now we operate with a realistic 3D model for the distribution of the magnetic field from the solar surface to the satellite. The perturbation of magnetic field lines serves as a string of a harp to deliver the information about the solar turbulence from the photosphere to the magnetometer. Owing to the propagation of Alfvén waves we can "listen" to the sounds of the great solar symphony. Due to absorption of the high frequency modes, however, at the transition region where the temperature jump occurs we are able to hear only the basses, whereas for the ultra-violin band we remain absolutely deaf. A current problem which deserves to be put on the agenda is to observe the powerful high frequency Alfvén modes under the corona. 23 A kamikaze satellite could give some very important information, but for systematic research we need to learn how to extract the behavior of the solar surface as a random driver using optical data. We conclude that the first important step in this direction is to establish correspondence between satellite magnetometric data and Doppler shift spectra for some bright events on the solar surface. Only after a proper incorporation of these ingredients we can conclude that our understanding of heating mechanism of the solar corona is complete and we have disclosed a very important case of heating of space plasmas.

\section{Acknowledgments}

Support and fruitful discussions with D. Damianov, A. Rogava, T. Zakarashvili, and R. Erdélyi are highly appreciated.
* E-mail: mishonov@phys.uni-sofia.bg

$\dagger$ E-mail: martin.stoev@gmail.com

‡ E-mail: yanamaneva@gmail.com

1 M. J. Aschwanden, Physics of the Solar Corona: an Introduction with Problems and Solutions, 2nd ed., Springer Berlin (2006).

${ }^{2}$ R. Erdélyi et. al. (eds) Turbulence, Waves and Instabilities in the Solar Plasma in NATO Science Series II: Mathematics, Physics and Chemistry, Vol. 124, Kluwer, pp.1-388 (2003) [ISBN: 1-4020-1658-1].

3 C. H. Mandrini, P. Démoulin and J. A. Klimchuk, "Magnetic field and plasma scaling laws: their implications for coronal heating models", ApJ, 530 999-1015 (2000).

4 M.B. Larson in Keneth R. Lang, Sun Earth and Sky (Springer-Verlag, Berlin, 1995); http://solar.physics.montana.edu/YPOP/Spotlight/ SunInfo/transreg.html.

5 R. Erdélyi and S.P. James, "Can ion-damping help to form spicules? II Random driver", A\&A, 427, 1055-1064 (2004), Figs. 8 and 10.

6 L.F. Burlaga and W.H. Mish, J. Gephys. Res., 92, 1261 (1987); E. Marsch, "MHD Turbulence in the Solar Wind" in Physics and Chemistry in Space - Space and Solar Physics, Vol. 21, Series Editors: M.C.E. Huber et al., Physics of the Inner Heliosphere, Vol. 2, Editors: R. Schwenn and E. Marsch, Springer-Verlag Berlin (1991); Fig. 10.4.

7 L. Landau and E. Lifschitz, Course of Theoretical Physics, Vol. 10 Physical Kinetics, (Pergamon, New York, 1981), Chap. IV, Sec. 43, Eqs. (43.8-10).

8 L. Landau and E. Lifschitz, Course of Theoretical Physics, Vol. 8 Electrodynamics of Continuous Media (Pergamon, New York, 1993), Chap. VIII Sec. 69.

9 S. I. Braginskii, Rev. Plasma Phys., 1, 205 (1965).

10 R. Erdélyi and M. Goossens, "Resonant absorption of Alfven waves in coronal loops in visco-resistive MHD", A\&A, 294, pp. 575-586 (1995).
11 M. Goossens, An introduction to plasma astrophysics and magnetohydrodynamics, Kluwer London, p. 108 (2003); see Chap. 4, eq. (4.38) and explanations thereafter.

12 T. Mishonov and Y. Maneva, "Burgulence and Alfvén Waves Heating Mechanism of Solar Corona", astro-ph/0609609

13 T. Mishonov, M. Stoev and Maneva, "Theory of heating of hot magnetized plasma by ALfvén waves. Application for solar corona", astro-ph/0701554.

14 L. Ofman, J. M. Davila and R. S. Steinolfson, "Coronal heating by the resonant absorption of Alfven waves: The effect of viscous stress tensor", ApJ 421, p. 360-371 (1994).

15 E. Schatzman, Annales d'Astrophysique, 12, 203 (1949); M. Schwarzschild, Astrophysical Journal, 107, 1 (1948).

16 J.A. Ionson, "Resonant absorption of Alfvénic surface waves and the heating of solar coronal loops", ApJ, 226, 650-673 (1978).

17 P. Langevin, Comptes. Rendues Ac. Sci. Paris, 146, 530 (1908).

18 J.M. Burgers, Adv. in Appl. Mech. 1, 171 (1948).

19 A.M. Polyakov, "Turbulence without pressure", Phys. Rev. E 52, 61836188 (1995); hep-th/9506189

20 A.N. Kolmogorov, "The local structute of turbulence in incompressible viscous fluid for very large Reynolds numbers," Comptes Rendue (Doklady) Acad. Sci. URSS (N.S.) 30, 301-305 (1941).

21 E. R. Priest, D. W. Longcope and J. Heyvaerts, "Coronal Heating at Separators and Separatrices", ApJ, 624, pp. 1057-1071 (2005).

22 T.M. Mishonov, Y.G. Maneva, T.S. Hristov, "On the theory of MHD waves in a shear flow of a magnetized turbulent plasma", astro-ph/0507696.

23 L. Ofman, J.M. Davila, V.M. Nakariakov, et al., "Highfrequency Alfven waves in multi-ion coronal plasma: Observational implications", Journal Of Geophysical Research 110 (A9), A09102, 0148-0227 (2005). 